\title{
PARAMETRIC VIBRATION OF MULTILAYER PLATES OF COMPLEX SHAPE
}

\author{
L. V. Kurpa, O.S. Mazur, and V. V. Tkachenko
}

UDC 539.3

\begin{abstract}
We propose a numerical-analytic method for the investigation of parametric vibrations of the plates under the action of static and periodic loads applied in the middle plane. The method is used for the equations of motion of plates obtained within the framework of the classical theory. The developed approach is based on the application of the theory of $R$-functions and variational methods, which enables us to study plates of arbitrary geometric shapes with various boundary conditions. According to the proposed approach, we first find the subcritical state of the plate if it is not homogeneous. To construct the zones of dynamic instability, we use the method proposed by Bolotin. The results obtained with the help of the developed approach are compared with the available data. We solve a number of new problems for multilayer plates of complex geometric shapes with holes.
\end{abstract}

The investigation of parametric vibration of multilayer composite plates is an important urgent problem of the contemporary nonlinear mechanics. As a specific feature of this class of problems, we can mention possible loss of stability for certain values of the parameters of loading, which leads to undesired consequences and even to the fracture of structures. In view of the fact that actual elements of thin-walled structures usually have holes, notches, and, generally speaking, arbitrary geometric shapes, which substantially affects the subcritical state of the plate, it is necessary to develop universal algorithms capable of determination of the subcritical state. For the state-of-the-art of this problem, the reader is referred to the works by Sahu and Datta [10], Dash, Asha, and Sahu [7], Simitses [11], Ng, Lam, and Reddy [9], Nemeth [8], etc. By analyzing the cited works, one can make a conclusion that numerical-analytic approaches to the investigation of parametric vibrations of multilayer plates of complex geometric shapes and in the case of inhomogeneity of the plates are practically absent.

A method proposed in the present work can be regarded as a universal approach that enables one to take into account the subcritical state and investigate multilayer plates with complex shape in the plan. For one-layer isotropic and orthotropic plates, a similar approach based on the $R$-function theory and variational methods $(R F M)$ [5] was developed in [6]. The novelty of the present work is connected with the development of the $R F M$ for the investigation of stability of multilayer plates of symmetric structure. In this case, we write (in the analytic form) the expressions for the coefficients of the system of ordinary differential equations obtained by reduction of the original system of motion with the help of multimode approximation of the unknown functions.

\section{Mathematical Statement of the Problem}

Consider a multilayer plate with symmetric structure (Fig. 1) and constant thickness $h$, which is relatively small as compared with the other sizes of the plate. To state the problem, we use the classical geometrically nonlinear theory based on the Kirchhoff-Love hypotheses.

\footnotetext{
"Kharkiv Polytechnic Institute” National Technical University, Kharkiv, Ukraine.
}

Translated from Matematychni Metody ta Fizyko-Mekhanichni Polya, Vol. 56, No. 2, pp. 136-150, April-June, 2013. Original article submitted January 29, 2013. 
According to this theory, the strain components in the middle plane are determined as follows:

$$
\begin{gathered}
\varepsilon_{x}=\frac{\partial u}{\partial x}+\frac{1}{2}\left(\frac{\partial w}{\partial x}\right)^{2}, \quad \varepsilon_{y}=\frac{\partial v}{\partial y}+\frac{1}{2}\left(\frac{\partial w}{\partial y}\right)^{2}, \quad \varepsilon_{x y}=\frac{\partial u}{\partial y}+\frac{\partial v}{\partial x}+\frac{\partial w}{\partial x} \frac{\partial w}{\partial y}, \\
\chi_{x}=-\frac{\partial^{2} w}{\partial x^{2}}, \quad \chi_{x y}=-2 \frac{\partial^{2} w}{\partial x \partial y}, \quad \chi_{y}=-\frac{\partial^{2} w}{\partial y^{2}},
\end{gathered}
$$

where $u, v$, and $w$ are displacements of points of the middle plane in the directions of the $O x-, O y-$, and $\mathrm{Oz}$-axes, respectively.

We represent the dependences of forces $N_{x}, N_{y}$, and $N_{x y}$ and moments $M_{x}, M_{y}$, and $M_{x y}$ on strains in the matrix form:

$$
\{N\}=[\boldsymbol{C}] \cdot\{\varepsilon\}, \quad\{M\}=[\boldsymbol{D}] \cdot\{\chi\},
$$

where $\boldsymbol{C}$ and $\boldsymbol{D}$ are the following matrices of reduced stiffness coefficients:

$$
\boldsymbol{C}=\left[\begin{array}{lll}
C_{11} & C_{12} & C_{16} \\
C_{12} & C_{22} & C_{26} \\
C_{16} & C_{26} & C_{66}
\end{array}\right], \quad \boldsymbol{D}=\left[\begin{array}{ccc}
D_{11} & D_{12} & D_{16} \\
D_{12} & D_{22} & D_{26} \\
D_{16} & D_{26} & D_{66}
\end{array}\right] .
$$

Denote by $\{\varepsilon\},\{\chi\},\{N\}$, and $\{M\}$ vectors of strains, forces, and moments, respectively,

$$
\begin{gathered}
\{\varepsilon\}=\left(\varepsilon_{x}, \varepsilon_{y}, \varepsilon_{x y}\right)^{\top}, \quad\{\chi\}=\left(\chi_{x}, \chi_{y}, \chi_{x y}\right)^{\top}, \\
\{N\}=\left(N_{x}, N_{y}, N_{x y}\right)^{\top}, \quad\{M\}=\left(M_{x}, M_{y}, M_{x y}\right)^{\top} .
\end{gathered}
$$

The reduced stiffness coefficients $C_{i j}$ and $D_{i j}(i j=11,22,12,16,26,66)$ are determined as follows [1]:

$$
\left(C_{i j}, D_{i j}\right)=\sum_{s=1}^{N} \int_{h_{s}}^{h_{s+1}} B_{i j}^{(s)}\left(1, z^{2}\right) d z, \quad i, j=1,2,6,
$$

where $B_{i j}^{(s)}$ are the elastic constants of the $s$ th layer.

In the general case where the axes of anisotropy do not coincide with the directions of the $O x$ - and $O y$ axes, the elastic constants $B_{i j}^{(s)}, i, j=1,2,6$, of the $s$ th layer are determined by the formulas of transformation of rotation of the axes of anisotropy by an angle $\alpha$ [1].

For the sake of convenience, we represent the forces $\{N\}=\left(N_{x}, N_{y}, N_{x y}\right)^{\top}$ and strains $\{\varepsilon\}=\left(\varepsilon_{x}, \varepsilon_{y}, \varepsilon_{x y}\right)^{\top}$ as the sums of the linear and nonlinear components:

$$
\{N\}=\{N\}^{(L)}+\{N\}^{(N)}, \quad\{\varepsilon\}=\{\varepsilon\}^{(L)}+\{\varepsilon\}^{(N)},
$$


where

$$
\begin{gathered}
\{\varepsilon\}^{(L)}=\left(\varepsilon_{x}^{(L)}, \varepsilon_{y}^{(L)}, \varepsilon_{x y}^{(L)}\right)^{\top}=\left(\frac{\partial u}{\partial x}, \frac{\partial v}{\partial y}, \frac{\partial u}{\partial y}+\frac{\partial v}{\partial x}\right)^{\top}, \\
\{\varepsilon\}^{(N)}=\left(\varepsilon_{x}^{(N)}, \varepsilon_{y}^{(N)}, \varepsilon_{x y}^{(N)}\right)^{\top}=\left(\frac{1}{2}\left(\frac{\partial w}{\partial x}\right)^{2}, \frac{1}{2}\left(\frac{\partial w}{\partial y}\right)^{2}, \frac{\partial w}{\partial y} \frac{\partial w}{\partial x}\right)^{\top} .
\end{gathered}
$$

Hence,

$$
\{N\}^{(L)}=[\boldsymbol{C}] \cdot\{\varepsilon\}^{(L)}, \quad\{N\}^{(N)}=[\boldsymbol{C}] \cdot\{\varepsilon\}^{(N)} .
$$

Neglecting the inertia forces in the middle plane, we represent the equation of motion in the operator form

$$
\begin{gathered}
L_{11} u+L_{12} v=-N \ell_{1}(w), \\
L_{21} u+L_{22} v=-N \ell_{2}(w), \\
L_{33} w=N \ell_{3}(u, v, w)-\varepsilon m_{1} \frac{\partial w}{\partial t}-m_{1} \frac{\partial^{2} w}{\partial t^{2}},
\end{gathered}
$$

where the operators $L_{i j}, N \ell_{i}, i, j=1,2,3$, are determined as follows:

$$
\begin{gathered}
L_{11}=C_{11} \frac{\partial^{2}}{\partial x^{2}}+2 C_{12} \frac{\partial^{2}}{\partial x \partial y}+C_{66} \frac{\partial^{2}}{\partial y^{2}} \\
L_{22}=C_{66} \frac{\partial^{2}}{\partial x^{2}}+2 C_{26} \frac{\partial^{2}}{\partial x \partial y}+C_{22} \frac{\partial^{2}}{\partial y^{2}} \\
L_{12}=L_{21}=C_{16} \frac{\partial^{2}}{\partial x^{2}}+\left(C_{12}+C_{66}\right) \frac{\partial^{2}}{\partial x \partial y}+C_{26} \frac{\partial^{2}}{\partial y^{2}}, \\
L_{33}=D_{11} \frac{\partial^{4}}{\partial x^{4}}+2\left(D_{12}+2 D_{66}\right) \frac{\partial^{4}}{\partial y^{2} \partial x^{2}}+4 D_{16} \frac{\partial^{4}}{\partial x^{3} \partial y}+4 D_{26} \frac{\partial^{4}}{\partial y^{3} \partial x}+D_{22} \frac{\partial^{4}}{\partial y^{4}} \\
N \ell_{1}=\frac{\partial w}{\partial x} L_{11} w+\frac{\partial w}{\partial y} L_{12} w \\
N \ell_{2}=\frac{\partial w}{\partial x} L_{12} w+\frac{\partial w}{\partial y} L_{22} w
\end{gathered}
$$




$$
N \ell_{3}=N_{x} \frac{\partial^{2} w}{\partial x^{2}}+N_{y} \frac{\partial^{2} w}{\partial y^{2}}+2 N_{x y} \frac{\partial^{2} w}{\partial x \partial y}
$$

In Eq. (3), $\varepsilon$ is the damping coefficient.

We supplement the system of equations (1)-(3) with the corresponding boundary conditions.

Note that, in the loaded part of the boundary, the imposed conditions in the plane of the plate have the form

$$
N_{n}=-p, \quad T_{n}=0,
$$

where

$$
N_{n}=N_{x} \ell^{2}+N_{y} m^{2}+2 N_{x y} \ell m, \quad T_{n}=N_{x y}\left(\ell^{2}-m^{2}\right)+\left(N_{y}-N_{x}\right) \ell m
$$

Here, $\ell$ and $m$ are the direction cosines of the outer normal $n$ to the boundary of the domain.

We also represent the contour forces $N_{n}$ and $T_{n}$ in the form of the sum of linear and nonlinear parts:

$$
N_{n}=N_{n}^{(L)}+N_{n}^{(N)}, \quad T_{n}=T_{n}^{(L)}+T_{n}^{(N)},
$$

where

$$
\begin{array}{ll}
N_{n}^{(L)}=\{P\} \cdot\{\varepsilon\}^{(L)}, & N_{n}^{(N)}=\{P\} \cdot\{\varepsilon\}^{(N)}, \\
T_{n}^{(L)}=\{Q\} \cdot\{\varepsilon\}^{(L)}, & T_{n}^{(N)}=\{Q\} \cdot\{\varepsilon\}^{(N)} .
\end{array}
$$

Here, by $\{P\}=\left(P_{1}, P_{2}, P_{3}\right)$ and $\{Q\}=\left(Q_{1}, Q_{2}, Q_{3}\right)$ we denote vectors with the following components:

$$
\begin{gathered}
P_{1}=C_{11} \ell^{2}+C_{12} m^{2}+2 C_{16} \ell m, \\
P_{2}=C_{12} \ell^{2}+C_{22} m^{2}+2 C_{26} \ell m, \\
P_{3}=C_{16} \ell^{2}+C_{26} m^{2}+2 C_{66} \ell m, \\
Q_{1}=C_{11}\left(\ell^{2}-m^{2}\right)+\left(C_{12}-C_{11}\right) \ell m, \\
Q_{2}=C_{26}\left(\ell^{2}-m^{2}\right)+\left(C_{22}-C_{12}\right) \ell m, \\
Q_{3}=C_{66}\left(\ell^{2}-m^{2}\right)+\left(C_{26}-C_{16}\right) \ell m .
\end{gathered}
$$

\section{Method for the Solution}

We now describe the algorithm developed for the solution of the problem of parametric vibrations of a multilayer plate as the following sequence of successive steps: 
$\mathbf{1}^{\circ}$. Determination of the inhomogeneous subcritical state of the plate.

$2^{\circ}$. Determination of the critical load.

$3^{\circ}$. Solution of the problem of linear vibrations of a plate loaded in its middle plane.

$4^{\circ}$. Solution of the nonlinear problem of vibrations of the plate.

$5^{\circ}$. Investigation of nonlinear vibrations of the plates compressed by static loads and construction of the backbone curves.

$6^{\circ}$. Construction of the domains of dynamic instability and determination of the amplitudes of stationary vibrations in the zones of parametric resonance.

$\mathbf{1}^{\circ}$. To perform the analysis of subcritical state, we solve the linearized system of the first two equations of motion (1), (2):

$$
\begin{aligned}
& L_{11} u_{1}+L_{12} v_{1}=0, \\
& L_{21} u_{1}+L_{22} v_{1}=0
\end{aligned}
$$

supplemented, in the loaded part of the boundary $\partial \Omega_{1}$, with the boundary conditions

$$
N_{n}^{(L)}\left(u_{1}, v_{1}\right)=-1, \quad T_{n}^{(L)}\left(u_{1}, v_{1}\right)=0 .
$$

The variational statement of problem (4)-(6) is reduced to finding the minimum of the functional

$$
I\left(u_{1}, v_{1}\right)=\frac{1}{2} \iint_{\Omega}\left(N_{x}^{(L)} \varepsilon_{x}+N_{y}^{(L)} \varepsilon_{y}+N_{x y}^{(L)} \varepsilon_{x y}\right) d \Omega+\int_{\partial \Omega_{1}} N_{n}^{(L)}\left(u_{1} \ell+v_{1} m\right) d \Omega_{1} .
$$

The minimum of functional (7) is sought in the set of functions satisfying the main (kinematic) boundary conditions. The system of basis functions is constructed by using the $R$-function theory [3-5]. Thus, if the plate is immobile in its plane in the unloaded part of the boundary $\partial \Omega_{2}$, then the set of admissible functions is determined with the help of structural formulas

$$
u_{1}=\omega_{2} \Phi_{1}, \quad v_{1}=\omega_{2} \Phi_{2},
$$

where $\omega_{2}=0$ is the equation of a part of the boundary $\partial \Omega_{2}$ and $\Phi_{i}, i=1,2$, are the undetermined components of the structural formulas [3-5]. According to the $R F M$, to find $\Phi_{i}$, we represent these components in the form of expansions in a complete system of functions $\left\{\varphi_{k}^{(i)}\right\}$ :

$$
\Phi_{i}=\sum_{k=1}^{n} a_{k}^{(i)} \varphi_{k}^{(i)}
$$


Here, $\left\{a_{k}^{(i)}\right\}$ are unknown coefficients determined from the condition of stationarity of functional (7):

$$
\frac{\partial I}{\partial a_{k}^{(i)}}=0
$$

As a result of the solution of the posed problem, we find the functions $u_{1}$ and $v_{1}$ and the forces

$$
\left\{N_{0}\right\}^{(L)}=\left(N_{0 x}^{(L)}\left(u_{1}, v_{1}\right), N_{0 y}^{(L)}\left(u_{1}, v_{1}\right), N_{0 x y}^{(L)}\left(u_{1}, v_{1}\right)\right)^{\top}
$$

determined as follows:

$$
\left\{N_{0}\right\}^{(L)}=[\boldsymbol{C}] \cdot\left\{\varepsilon_{0}\right\}^{(L)},
$$

where

$$
\begin{gathered}
\left\{\varepsilon_{0}\right\}^{(L)}=\left(\varepsilon_{0 x}^{(L)}, \varepsilon_{0 y}^{(L)}, \varepsilon_{0 x y}^{(L)}\right)^{\top}, \\
\varepsilon_{0 x}^{(L)}=\frac{\partial u_{1}}{\partial x}, \quad \varepsilon_{0 y}^{(L)}=\frac{\partial v_{1}}{\partial y}, \quad \varepsilon_{0 x y}^{(L)}=\frac{\partial u_{1}}{\partial y}+\frac{\partial v_{1}}{\partial x} .
\end{gathered}
$$

$2^{\circ}$. To find the critical load, we consider Eq. (3) and take into account the fact that compressive loads are proportional to a certain parameter $\lambda$ :

$$
L_{33} w=\lambda \cdot N \ell_{3}\left(u_{1}, v_{1}, w\right)
$$

We determine the critical value of the parameter $\lambda$ by using the energy approach according to which the solution of Eq. (9) with the corresponding boundary conditions can be reduced to an equivalent variational problem of finding the minimum of the following functional:

$$
I(w)=\frac{1}{2} \iint_{\Omega}\left[\left(M_{x} \chi_{x}+M_{y} \chi_{y}+M_{x y} \chi_{x y}\right)+\lambda\left(N_{x}^{0}\left(\frac{\partial w}{\partial x}\right)^{2}+N_{y}^{0}\left(\frac{\partial w}{\partial y}\right)^{2}+N_{x y}^{0} \frac{\partial w}{\partial x} \frac{\partial w}{\partial y}\right)\right] d x d y .
$$

As above, the minimization of functional (10) is performed in the set of the basis functions constructed with the help of the RFM.

Thus, the structure of the solution satisfying the conditions of clamped fixing has the form

$$
w=\omega^{2} \Phi_{3},
$$

where $\Phi_{3}$ is the undetermined component of the structure, which must be expanded in a series with respect to a certain complete system of functions. For the other types of boundary conditions, the corresponding structures are presented in [3]. 
As a result of application of the Ritz method, the problem is reduced to the eigenvalue problem. The smallest eigenvalue determines the value of the critical parameter $\lambda$ and, hence, the critical load.

$3^{\circ}$. The solution of the problem of linear vibrations of plates subjected to the action of a static load $p_{0}$ is also obtained by the Ritz method as a result of the minimization of the following functional:

$$
I=\Pi_{\max }-\mathrm{T}_{\max },
$$

where $\Pi_{\max }$ is the total potential energy of the plate taking into account the influence of forces acting in the middle plane

$$
\begin{aligned}
\Pi_{\max }=\frac{1}{2} \iint_{\Omega}[ & M_{x} \chi_{x}+M_{y} \chi_{y}+M_{x y} \chi_{x y} \\
& \left.+p_{0}\left(N_{x}^{(L)}\left(u_{1}, v_{1}\right)\left(\frac{\partial w}{\partial x}\right)^{2}+N_{y}^{(L)}\left(u_{1}, v_{1}\right)\left(\frac{\partial w}{\partial y}\right)^{2}+N_{x y}^{(L)}\left(u_{1}, v_{1}\right) \frac{\partial w}{\partial x} \frac{\partial w}{\partial y}\right)\right] d \Omega
\end{aligned}
$$

and $\mathrm{T}_{\max }$ is the kinetic energy of the plate

$$
\mathrm{T}_{\max }=\frac{m_{1} \Omega_{L}^{2}}{2} \iint_{\Omega} w^{2} d \Omega,
$$

where $\Omega_{L}$ is the natural frequency that corresponds to the load $p_{0}$.

$4^{\circ}$. To solve the problem of geometrically nonlinear vibrations of the compressed plate, we represent the unknown functions $(u, v, w)$ in the form

$$
\begin{gathered}
w(x, y, t)=\sum_{i=1}^{n} y_{i}(t) w_{i}(x, y), \\
u(x, y, t)=u_{1}(x, y) p+\sum_{i, j=1}^{n} y_{i}(t) y_{j}(t) u_{i j}(x, y), \\
v(x, y, t)=v_{1}(x, y) p+\sum_{i, j=1}^{n} y_{i}(t) y_{j}(t) v_{i j}(x, y),
\end{gathered}
$$

where $p$ is a load determined as follows:

$$
p=p_{0}+p_{t} \cos \theta t
$$


$p_{0}$ is the static component of the load, $p_{t}$ is the amplitude of the periodic part of the load, $w_{i}(x, y)$ are eigenfunctions corresponding to certain frequencies of linear vibrations of the plate under the action of the load $p_{0}$, and the functions $\left(u_{i j}, v_{i j}\right)$ are the solutions of the system

$$
\begin{aligned}
& L_{11}\left(u_{i j}\right)+L_{12}\left(v_{i j}\right)=-N \ell_{1}^{(2)}\left(w_{i}, w_{j}\right), \\
& L_{21}\left(u_{i j}\right)+L_{22}\left(v_{i j}\right)=-N \ell_{2}^{(2)}\left(w_{i}, w_{j}\right),
\end{aligned}
$$

supplemented with the following boundary conditions on the loaded part:

$$
\begin{aligned}
& N_{n}^{(L)}\left(u_{i j}, v_{i j}\right)=-N_{n}^{(N)}\left(w_{i}, w_{j}\right), \\
& T_{n}^{(L)}\left(u_{i j}, v_{i j}\right)=-T_{n}^{(N)}\left(w_{i}, w_{j}\right) .
\end{aligned}
$$

On the other parts of the boundary, the geometric boundary conditions for the functions $\left(u_{i j}, v_{i j}\right)$ are the same as in the linear problem of step 1 and the static conditions take the form (13).

The right-hand sides of Eqs. (12) are determined as follows:

$$
\begin{aligned}
& N \ell_{1}^{(2)}\left(w_{i}^{(c)}, w_{j}^{(c)}\right)=w_{i, x} L_{11} w_{j}+w_{i, y} L_{12} w_{j}, \\
& N \ell_{2}^{(2)}\left(w_{i}^{(c)}, w_{j}^{(c)}\right)=w_{i, x} L_{12} w_{j}+w_{i, y} L_{22} w_{j} .
\end{aligned}
$$

For the right-hand sides of the boundary conditions (13), we can write

$$
\begin{aligned}
& N_{n}^{(N)}\left(w_{i}, w_{j}\right)=\frac{1}{2}\left(P_{1} \frac{\partial w_{i}}{\partial x} \frac{\partial w_{j}}{\partial x}+P_{2} \frac{\partial w_{i}}{\partial y} \frac{\partial w_{j}}{\partial y}+P_{3}\left(\frac{\partial w_{i}}{\partial y} \frac{\partial w_{j}}{\partial x}+\frac{\partial w_{i}}{\partial x} \frac{\partial w_{j}}{\partial y}\right)\right) \\
& T_{n}^{(N)}\left(w_{i}, w_{j}\right)=\frac{1}{2}\left(Q_{1} \frac{\partial w_{i}}{\partial x} \frac{\partial w_{j}}{\partial x}+Q_{2} \frac{\partial w_{i}}{\partial y} \frac{\partial w_{j}}{\partial y}+Q_{3}\left(\frac{\partial w_{i}}{\partial y} \frac{\partial w_{j}}{\partial x}+\frac{\partial w_{i}}{\partial x} \frac{\partial w_{j}}{\partial y}\right)\right)
\end{aligned}
$$

The variational statement of problem (12), (13) is reduced to finding the minimum of the functional

$$
\begin{aligned}
I\left(u_{i j}, v_{i j}\right)=\frac{1}{2} \iint_{\Omega}\left(N_{x}^{(L)} \varepsilon_{x}^{(L)}\right. & +N_{y}^{(L)} \varepsilon_{y}^{(L)}+N_{x y}^{(L)} \varepsilon_{x y}^{(L)}-2\left(N \ell_{1}^{(2)}\left(w_{i}, w_{j}\right) u_{i j}\right. \\
& \left.\left.+N \ell_{2}^{(2)}\left(w_{i}, w_{j}\right) v_{i j}\right)\right) d \Omega+\int_{\partial \Omega} N_{n}^{(N)}\left(w_{i}, w_{j}\right)\left(u_{i j} \ell+v_{i j} m\right) \\
& +T_{n}^{(N)}\left(w_{i}, w_{j}\right)\left(-u_{i j} m+v_{i j} \ell\right) d \Omega_{1} .
\end{aligned}
$$


As above, we solve the sequence of problems (14) by the Ritz variational method. The systems of the basis functions are constructed by using the RFM.

It is easy to see that, after the substitution of expressions (11) in the initial system (1)-(3), the first two equations are identically satisfied and Eq. (3) takes the form

$$
\begin{aligned}
\sum_{k=1}^{n} y_{k}(t) L_{33} w_{k}= & -m_{1}\left(\sum_{k=1}^{n}\left(y_{k}^{\prime \prime}(t)+\varepsilon y_{k}^{\prime}(t)\right) w_{k}(x, y)\right) \\
& +p(t) \sum_{k=1}^{n} y_{k}(t) N \ell_{31}\left(u_{1}, v_{1}, w_{1}\right)+\sum_{k=1}^{n} \sum_{i=1}^{n} \sum_{j=1}^{n} y_{i}(t) y_{j}(t) y_{k}(t) N \ell_{32}\left(u_{i j}, v_{i j}, w_{k}\right),
\end{aligned}
$$

where

$$
\begin{gathered}
N \ell_{31}\left(u_{1}, v_{1}, w_{k}\right)=N_{0 x}^{(L)} \frac{\partial^{2} w_{k}}{\partial x^{2}}+N_{0 y}^{(L)} \frac{\partial^{2} w_{k}}{\partial y^{2}}+2 N_{0 x y}^{(L)} \frac{\partial^{2} w_{k}}{\partial x \partial y}, \\
N \ell_{32}\left(u_{i j}, v_{i j}, w_{k}\right)=N_{x}^{(N p)}\left(u_{i j}, v_{i j}, w_{k}\right) \frac{\partial^{2} w_{k}}{\partial x^{2}}+N_{y}^{(N p)}\left(u_{i j}, v_{i j}, w_{k}\right) \frac{\partial^{2} w_{k}}{\partial y^{2}}+2 N_{x y}^{(N p)}\left(u_{i j}, v_{i j}, w_{k}\right) \frac{\partial^{2} w_{k}}{\partial x \partial y} .
\end{gathered}
$$

In this case, $N_{0 x}^{(L)}, N_{0 y}^{(L)}$, and $N_{0 x y}^{(L)}$ are determined by relations (8) and $N_{x}^{(N p)}, N_{y}^{(N p)}, N_{x y}^{(N p)}$ take the following form:

$$
\{N\}^{(N p)}=\left[\begin{array}{c}
N_{x}^{(N p)} \\
N_{y}^{(N p)} \\
N_{x y}^{(N p)}
\end{array}\right]=[\boldsymbol{C}]\left[\begin{array}{c}
\frac{\partial u_{i j}}{\partial x}+\frac{1}{2} \frac{\partial w_{i}}{\partial x} \frac{\partial w_{j}}{\partial x} \\
\frac{\partial v_{i j}}{\partial x}+\frac{1}{2} \frac{\partial w_{i}}{\partial y} \frac{\partial w_{j}}{\partial y} \\
\frac{\partial u_{i j}}{\partial y}+\frac{\partial v_{i j}}{\partial x}+\frac{1}{2}\left(\frac{\partial w_{i}}{\partial x} \frac{\partial w_{j}}{\partial y}+\frac{\partial w_{i}}{\partial x} \frac{\partial w_{j}}{\partial y}\right)
\end{array}\right]
$$

In view of the fact that $p=p_{0}+p_{t} \cos \theta t$, we represent Eq. (15) in the form

$$
\begin{aligned}
& \sum_{k=1}^{n} y_{k}(t)\left(L_{33} w_{k}-p_{0} N \ell_{31}\left(u_{1}, v_{1}, w_{k}\right)\right) \\
& =p_{t} \cos \theta t \sum_{k=1}^{n} y_{k}(t) N \ell_{31}\left(u_{1}, v_{1}, w_{k}\right) \\
& \quad-m_{1} \sum_{k=1}^{n}\left(y_{k}^{\prime \prime}(t)+\varepsilon y_{k}^{\prime}(t) w_{k}(x, y)\right)+\sum_{k=1}^{n} \sum_{i=1}^{n} \sum_{j=1}^{n} y_{i}(t) y_{j}(t) y_{k}(t) N \ell_{32}\left(u_{i j}, v_{i j}, w_{k}\right) .
\end{aligned}
$$


Note that the expression in parentheses on the left-hand side in the first sum is equal to $m_{1} \Omega_{k}^{2} w_{k}$. Applying the Bubnov-Galerkin method to Eq. (16) for the orthogonal eigenfunctions $w_{m}, m=1, \ldots, n$, and dividing the result by $m_{1}\left\|w_{m}\right\|^{2}$, we arrive at the system of equations

$$
y_{m}^{\prime \prime}(t)+\varepsilon y_{m}^{\prime}(t)+\Omega_{m}^{2}\left(y_{m}+p_{t} \cos \theta t \sum_{k=1}^{n} \alpha_{k}^{(m)} y_{k}+\sum_{i=1}^{n} \sum_{j=1}^{n} \sum_{k=1}^{n} \gamma_{i j k}^{(m)} y_{i}(t) y_{j}(t) y_{k}(t)\right)=0, \quad m=1, \ldots, n,
$$

where the coefficients of the system of equations (17) are given by the following formulas:

$$
\begin{array}{r}
\alpha_{k}^{(m)}=-\frac{\iint_{\Omega} N \ell_{31}\left(u_{1}, v_{1}, w_{k}\right) w_{m} d \Omega}{m_{1} \Omega_{m}^{2}\left\|w_{m}\right\|^{2}}, \\
\gamma_{i j k}^{(m)}=-\frac{\iint_{\Omega} N \ell_{32}\left(u_{i j}, v_{i j}, w_{k}\right) w_{m} d \Omega}{m_{1} \Omega_{m}^{2}\left\|w_{m}\right\|^{2}} .
\end{array}
$$

The investigation of system (17) is a complicated problem but if we restrict ourselves to the single-mode approximation of the unknown functions, i.e., represent the deflection of the plate in the form

$$
w(x, y, t)=y(t) w_{1}(x, y),
$$

and displacements in the middle plane in the form

$$
\begin{aligned}
& u(x, y, t)=p(t) u_{1}(x, y)+y^{2}(t) \cdot u_{11}(x, y), \\
& v(x, y, t)=p(t) v_{1}(x, y)+y^{2}(t) \cdot v_{11}(x, y),
\end{aligned}
$$

then the system of equations (17) is reduced to a single equation

$$
y^{\prime \prime}(t)+\varepsilon y^{\prime}(t)+\Omega_{L}^{2}\left(1+\alpha p_{t} \cos \theta t+\gamma y^{2}(t)\right) y(t)=0,
$$

where $y(t)=y_{1}(t), \Omega_{L}=\Omega_{1}$, and the coefficients $\alpha=\alpha_{1}^{(1)}$ and $\gamma=\gamma_{111}^{(1)}$ are given by the formulas

$$
\begin{aligned}
& \alpha=-\frac{\iint_{\Omega} N \ell_{31}\left(u_{1}, v_{1}, w_{1}\right) w_{1} d \Omega}{m_{1} \Omega_{L}^{2}\left\|w_{1}\right\|^{2}}, \\
& \gamma=-\frac{\iint_{\Omega} N \ell_{32}\left(u_{1}, v_{1}, w_{1}\right) w_{1} d \Omega}{m_{1} \Omega_{L}^{2}\left\|w_{1}\right\|^{2}} .
\end{aligned}
$$


We denote the expression $\alpha \cdot p_{t}=-2 k, \varepsilon_{1}=\varepsilon / 2$. Then Eq. (18) takes the same form as in [2]:

$$
y^{\prime \prime}(t)+2 \varepsilon_{1} y^{\prime}(t)+\Omega_{L}^{2}\left((1-2 k \cos \theta t) y(t)+\gamma y^{3}(t)\right)=0 .
$$

$5^{\circ}$. Under the static load, we have $p_{t}=0$, whence $k=0$. Then the investigation is reduced to the solution of the following equation:

$$
y^{\prime \prime}(t)+\Omega_{L}^{2}\left(y(t)+\gamma y^{3}(t)\right)=0
$$

(here, $\varepsilon=0$ ). The application of the Bubnov-Galerkin method to this equation enables us to obtain the dependence of the amplitude of nonlinear vibrations on the ratio of the nonlinear frequency to the linear frequency $v=\omega_{N} / \Omega_{L}$ :

$$
v=\sqrt{1+\frac{3}{4} \gamma A^{2}}
$$

$6^{\circ}$. In the case where periodic load acts upon the plate, it is necessary to determine the zones of dynamic instability of the system. To investigate its stability, as shown in [2], it suffices to consider the linearized equation (19), i.e., for $\gamma=0$,

$$
y^{\prime \prime}(t)+2 \varepsilon_{1} y^{\prime}(t)+\Omega_{L}^{2}(1-2 k \cdot \cos \theta t) y(t)=0 .
$$

It is known that, for Eq. (20) (Mathieu equation), the main domain of instability (near $\theta=2 \Omega_{L}$ ) is bounded by the following curves [2]:

$$
2 \Omega_{L} \sqrt{1-\sqrt{k^{2}-\left(\frac{\Delta}{\pi}\right)^{2}}} \leq \theta \leq 2 \Omega_{L} \sqrt{1+\sqrt{k^{2}-\left(\frac{\Delta}{\pi}\right)^{2}}}
$$

where by $\Delta=\frac{2 \pi \varepsilon_{1}}{\Omega}$ we denote the damping decrement [2]. For $\varepsilon_{1}=0$, we get

$$
\theta_{1}=2 \Omega_{L} \sqrt{1-k}, \quad \theta_{2}=2 \Omega_{L} \sqrt{1+k}
$$

In this interval, we observe the main parametric resonance.

To find the amplitudes of stationary vibrations in the zone of the principal parametric resonance, we use the approach presented in [2] and obtain the following relationship for the frequency ratio $\theta / 2 \Omega_{L}$ and the amplitude of vibrations $A$ :

$$
A=\frac{2}{\sqrt{3 \gamma}} \sqrt{\left(\frac{\theta}{2 \Omega_{L}}\right)^{2}-1 \pm \sqrt{k^{2}-\left(\frac{\theta}{2 \Omega_{L}}\right)^{2}\left(\frac{\Delta}{\pi}\right)^{2}}} .
$$




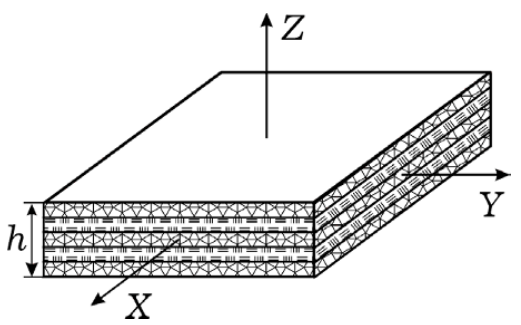

(a)



(b)

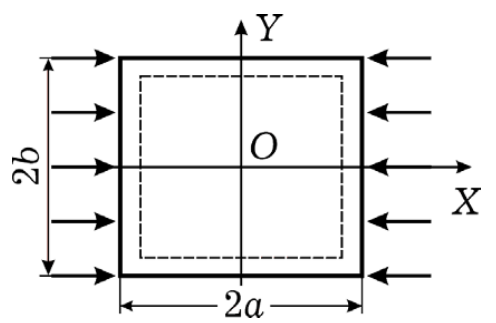

(c)

Fig. 1

Table 1

\begin{tabular}{|c|c|c|c|c|c|c|}
\hline \multirow[b]{3}{*}{$A$} & \multicolumn{6}{|c|}{ Boundary conditions } \\
\hline & \multicolumn{2}{|c|}{ Simply supported (21) } & \multicolumn{2}{|c|}{ Simply supported (22) } & \multicolumn{2}{|c|}{ Clamped (23) } \\
\hline & $R F M$ & [12] & $R F M$ & [12] & $R F M$ & {$[12]$} \\
\hline \multicolumn{7}{|c|}{$\left[0^{\circ} / 90^{\circ} / 0^{\circ} / 90^{\circ} / 0^{\circ}\right]$} \\
\hline 0.2 & 1.031 & 1.031 & 1.001 & 1.001 & 1.008 & 1.008 \\
\hline 0.4 & 1.120 & 1.121 & 1.006 & 1.006 & 1.031 & 1.033 \\
\hline 0.6 & 1.254 & 1.257 & 1.013 & 1.015 & 1.068 & 1.073 \\
\hline 0.8 & 1.420 & 1.428 & 1.024 & 1.026 & 1.119 & 1.128 \\
\hline 1.0 & 1.609 & 1.624 & 1.037 & 1.040 & 1.180 & 1.194 \\
\hline 1.2 & 1.813 & 1.837 & 1.053 & 1.057 & 1.251 & 1.271 \\
\hline \multicolumn{7}{|c|}{$\left[45^{\circ} /-45^{\circ} / 45^{\circ} /-45^{\circ} / 45^{\circ}\right]$} \\
\hline 0.2 & 1.014 & 1.015 & 1.008 & 1.008 & 1.007 & 1.007 \\
\hline 0.4 & 1.057 & 1.058 & 1.031 & 1.031 & 1.028 & 1.029 \\
\hline 0.6 & 1.125 & 1.126 & 1.069 & 1.069 & 1.061 & 1.063 \\
\hline 0.8 & 1.214 & 1.215 & 1.119 & 1.118 & 1.105 & 1.109 \\
\hline 1.0 & 1.319 & 1.322 & 1.181 & 1.178 & 1.161 & 1.165 \\
\hline 1.2 & 1.436 & 1.442 & 1.252 & 1.246 & 1.225 & 1.230 \\
\hline
\end{tabular}

\section{Testing of the Proposed Approach}

To test the proposed algorithm, we consider vibrations of a five-layer square plate (Fig. 1) with different types of boundary conditions: 
- simply supported plate immovable in its plane

$$
u_{n}=0, \quad v_{n}=0, \quad w=0, \quad M_{n}=0
$$

- simply supported plate partially movable in its plane

$$
u_{n}=0, \quad T_{n}=0, \quad w=0, \quad M_{n}=0 ;
$$

- clamped plate

$$
u_{n}=0, \quad v_{n}=0, \quad w=0, \quad \frac{\partial w}{\partial n}=0
$$

We performed investigations for a plate with the following properties of the material: $E_{1} / E_{2}=40$, $G / E_{2}=0.6$, and $v_{1}=0.25$. Assume that all layers have the same thickness and the total thickness of the plate satisfies the relation $h / a=0.01$.

Note that, in the considered case, the subcritical state of the plate is homogeneous, and hence, step $1^{\circ}$ could be omitted but, to check the developed software, this step was also realized.

In Table 1, we present the comparison of values of the frequency ratio $v=\omega_{N} / \omega_{L} \quad\left(\omega_{L}\right.$ is the natural frequency of linear vibrations of the unloaded plate) under different boundary conditions for the five-layer square plate, different amplitudes $A$, and two methods of stacking of the layers. The difference between the obtained results and the results presented in [12] does not exceed $3 \%$.

\section{Investigation of Parametric Vibrations of a Complex-Shaped Plate}

Consider a five-layer plate with symmetric structure and the following way of stacking of the layers: $\left[0^{\circ} / 90^{\circ} / 0^{\circ} / 90^{\circ} / 0^{\circ}\right]$ (see Fig. 2).

The shape of the plate is complicated by the corner cutouts and a hole in the inner part.

Assume that a load is applied along the sides parallel to the $O Y$-axis. We also assume that, on the outer boundary, the function of deflection satisfies the conditions of simple support $w=0$ and $M_{n}=0$ and the inner boundary is free. For the displacements in the plane of the plate in the loaded part of the boundary, the boundary conditions take the form

$$
N_{n}=-p, \quad T_{n}=0 .
$$

At the same time, in the unloaded part, the boundary conditions have the form

$$
N_{n}=0, \quad T_{n}=0 .
$$

The numerical investigations were performed for a plate with the following geometric parameters: $b / a=1$, $h / a=0.01$, and $c / a=0.35$. The size of the hole $r / a$ is varied. The properties of the considered material are presented in Table 2 .

We choose the structure of the solution as follows:

$$
u=\Phi_{1}, \quad v=\Phi_{2}, \quad w=\omega_{2} \Phi_{3},
$$




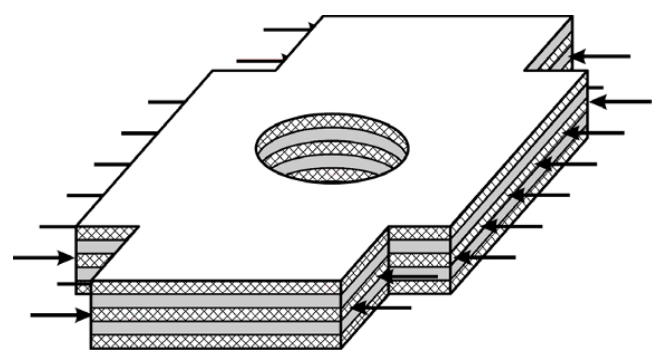

(a)

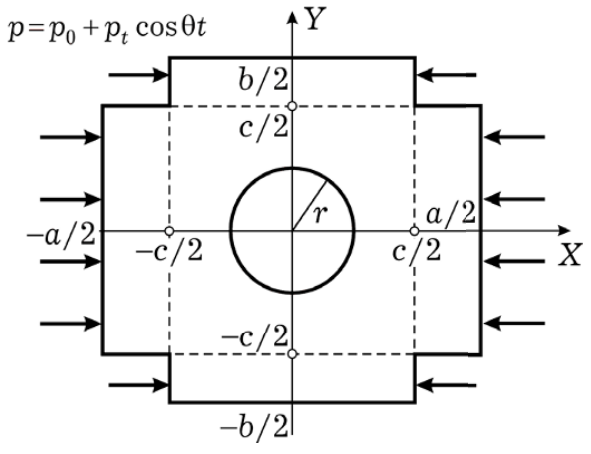

(b)

Fig. 2

Table 2

\begin{tabular}{ccccc}
\hline Material & $E_{1} / E_{2}$ & $G / E_{2}$ & $\mathrm{v}_{1}=v_{2} E_{1} / E_{2}$ \\
\hline Glass-epoxy & 3 & 0.6 & 0.25 \\
Boron-epoxy & 10 & 0.33 & 0.22 \\
Graphite & 40 & 0.6 & 0.25 \\
\hline
\end{tabular}

where $\omega_{2}(x, y)=0$ is the equation of the outer boundary of the domain,

$$
\omega_{2}(x, y)=f_{1} \wedge_{0} f_{2} \wedge_{0}\left(f_{3} \vee_{0} f_{4}\right) .
$$

The functions $f_{1}, f_{2}, f_{3}$, and $f_{4}$ have the form

$$
\begin{aligned}
& f_{1}=\frac{1}{a}\left(\left(\frac{a}{2}\right)^{2}-x^{2}\right) \geq 0, \quad f_{2}=\frac{1}{b}\left(\left(\frac{b}{2}\right)^{2}-y^{2}\right) \geq 0, \\
& f_{3}=\frac{1}{c}\left(\left(\frac{c}{2}\right)^{2}-x^{2}\right) \geq 0, \quad f_{4}=\frac{1}{c}\left(\left(\frac{c}{2}\right)^{2}-y^{2}\right) \geq 0 .
\end{aligned}
$$

The symbols $\vee_{0}$ and $\wedge_{0}$ in (24) determine the $R$-operations [5].

The values of the frequency parameter

$$
\Lambda_{i}=\Omega_{i} a^{2} \sqrt{\frac{12\left(1-\mu_{1} \mu_{2}\right)}{E_{2} h^{2}}}, i=1, \ldots, 4
$$

obtained for different materials for $r / a=0.15$ are presented in Table 3. The corresponding modes of vibrations for the graphite material are shown in Fig. 3. 


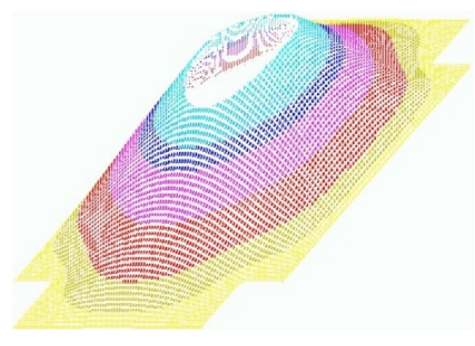

(a) $\Lambda_{1}=100.835$

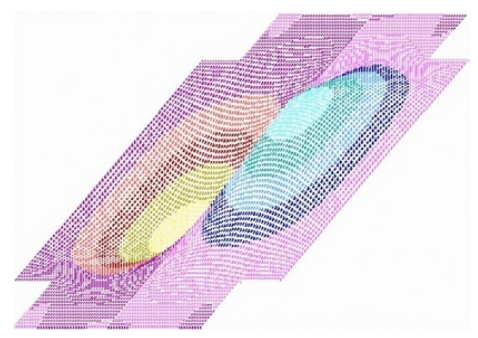

(c) $\Lambda_{3}=244.552$

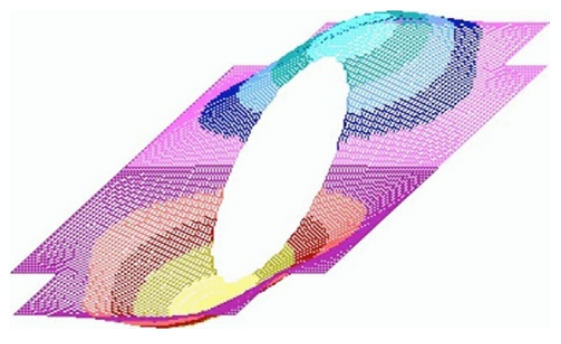

(b) $\Lambda_{2}=196.997$

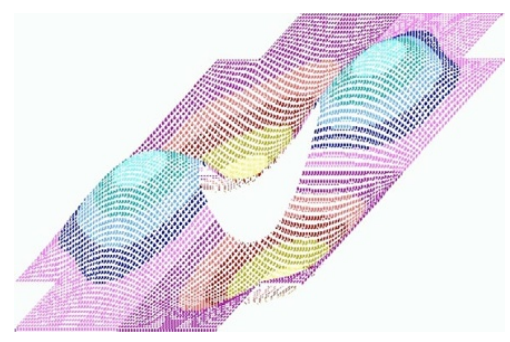

(d) $\Lambda_{4}=371.306$

Fig. 3

Table 3

\begin{tabular}{l|r|r|r|c}
\hline & $\Lambda_{1}$ & $\Lambda_{2}$ & $\Lambda_{3}$ & $\Lambda_{4}$ \\
\hline Glass-epoxy & 41.069 & 79.561 & 86.414 & 143.419 \\
Boron-epoxy & 55.157 & 108.418 & 128.919 & 202.165 \\
Graphite & 100.835 & 196.997 & 244.552 & 371.306 \\
\hline
\end{tabular}

The values of the frequency parameter

$$
\Lambda=\Omega_{L} a^{2} \sqrt{\frac{12\left(1-\mu_{1} \mu_{2}\right)}{E_{2} h^{2}}}
$$

obtained for different materials, different sizes of the hole $r / a$, and different values of the load $p_{0} / p_{k r}$ are presented in Table 4.

The data presented in Table 4 enable us to conclude that the frequency parameter $\Lambda$ decreases as the load $p_{0} / p_{k r}$ acting upon the plate (for given $r / a$ ) increases. At the same time, the frequency parameter increases as the size of the hole $r / a$ increases in the chosen interval $0.1 \leq r / a \leq 0.25$.

In Figs. 4-7, we show the domains of dynamic instability (DDI) for different types of materials, sizes of the hole $r / a$, values of the static load, and levels of damping. In Fig. 4, we present the DDI for a plate made of a glass-epoxy material for different $r / a$. The size of the hole strongly affects the location of the DDI. Thus, as the size increases, the domains of instability shift to higher values of the exciting frequency. 


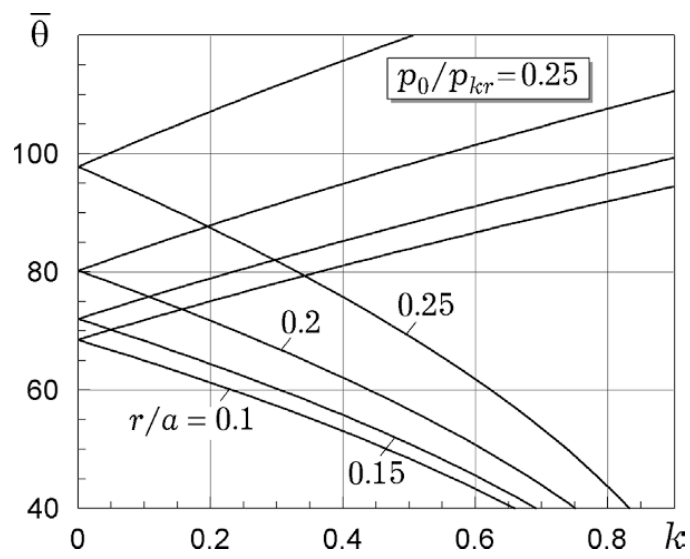

Fig. 4

Table 4

\begin{tabular}{c|c|c|c|c|c}
\hline \multirow{2}{*}{ Material } & \multirow{2}{*}{$r / a$} & \multicolumn{4}{|c}{$p_{0} / p_{k r}$} \\
\cline { 3 - 6 } & & 0 & 0.25 & 0.5 & 0.75 \\
\hline \multirow{2}{*}{ Glass-epoxy } & 0.1 & 39.275 & 34.261 & 28.228 & 20.194 \\
& 0.15 & 41.069 & 36.022 & 29.868 & 21.529 \\
& 0.25 & 53.446 & 48.904 & 42.797 & 33.927 \\
\hline \multirow{3}{*}{ Boron-epoxy } & 0.15 & 55.157 & 48.091 & 39.578 & 28.257 \\
& 0.2 & 59.817 & 52.474 & 43.461 & 31.241 \\
& 0.25 & 68.808 & 64.201 & 54.791 & 43.115 \\
\hline \multirow{4}{*}{ Graphite } & 0.1 & 99.030 & 86.016 & 70.474 & 50.039 \\
& 0.15 & 100.835 & 87.864 & 72.254 & 51.529 \\
& 0.2 & 109.007 & 95.347 & 78.720 & 56.416 \\
& 0.25 & 124.439 & 113.496 & 98.537 & 76.815 \\
\hline
\end{tabular}

Curves $1-3$ in Fig. 5 show the DDI for a hole with $r / a=0.2$ for $p_{0} / p_{k r}=0.5$ and for the glass-epoxy, boron-epoxy, and graphite materials, respectively.

In Fig. 6, we present the DDI for the boron-epoxy material with a hole $r / a=0.2$ for various values of the loading parameter $p_{0} / p_{k r}$. In Fig. 7, we display the DDI for the same material for various values of the damp- 


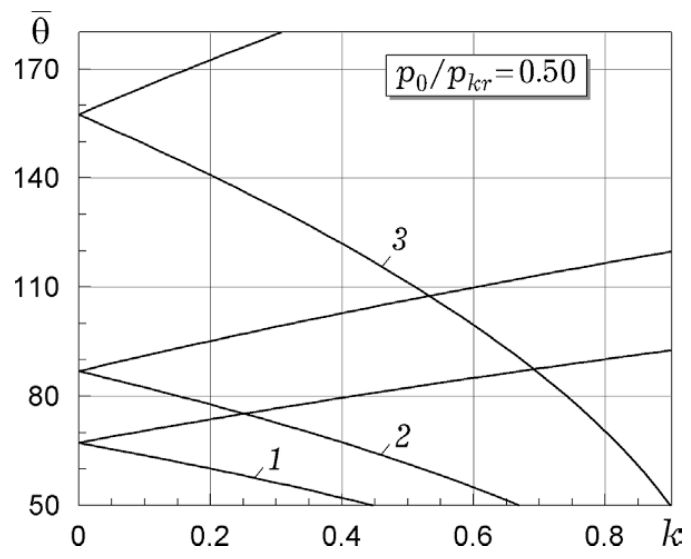

Fig. 5

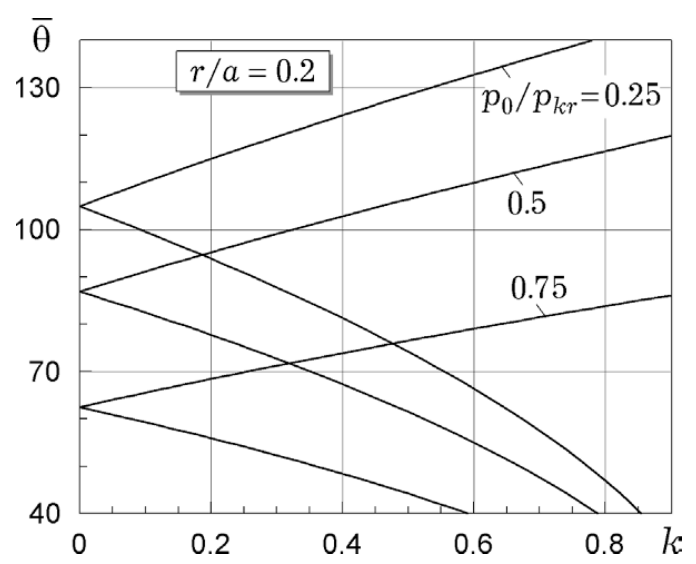

Fig. 6

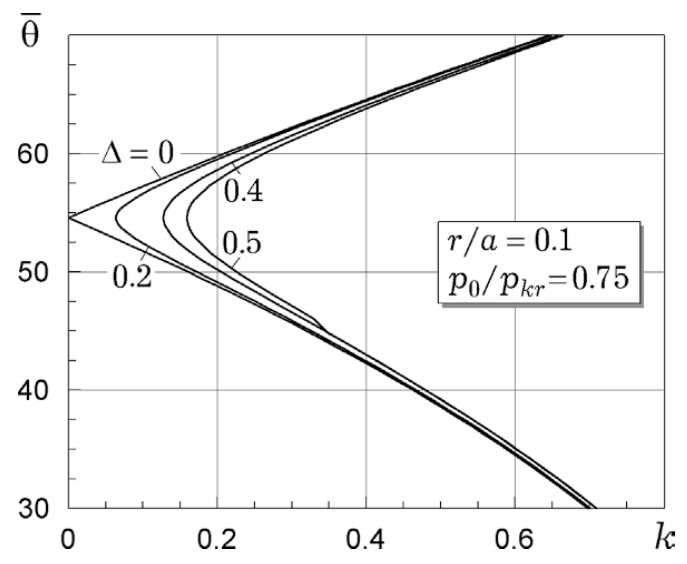

Fig. 7

ing decrement $\Delta$ and fixed $r / a=0.1$ and $p_{0} / p_{k r}=0.75$. Note that the loss of stability for the plates made of the graphite material appears for much higher values of the exciting frequency. As the constant component of the load $p_{0}$ increases, the domain of dynamic instability shifts to lower values of the exciting frequency. An increase in the damping decrement makes the DDI smaller. 


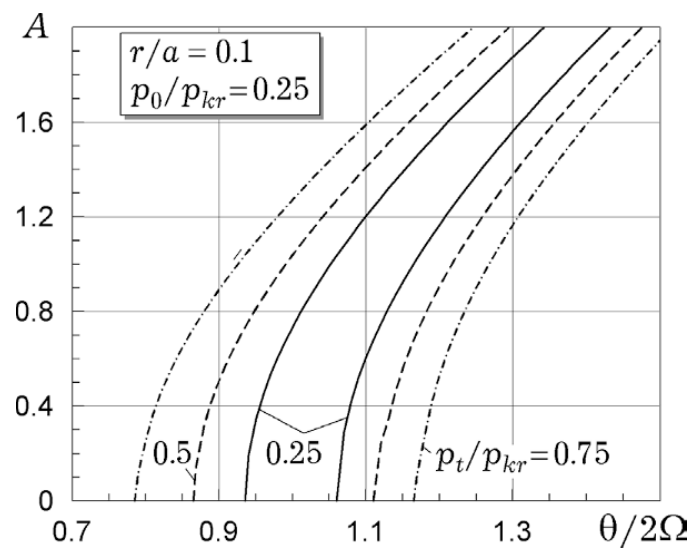

Fig. 8

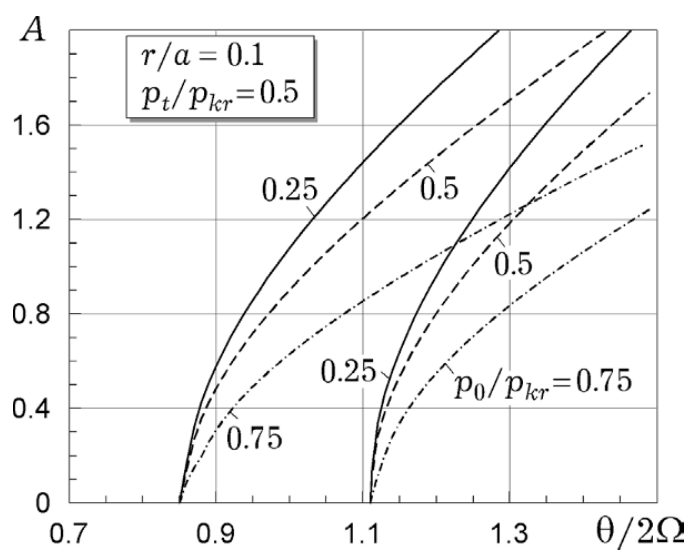

Fig. 9

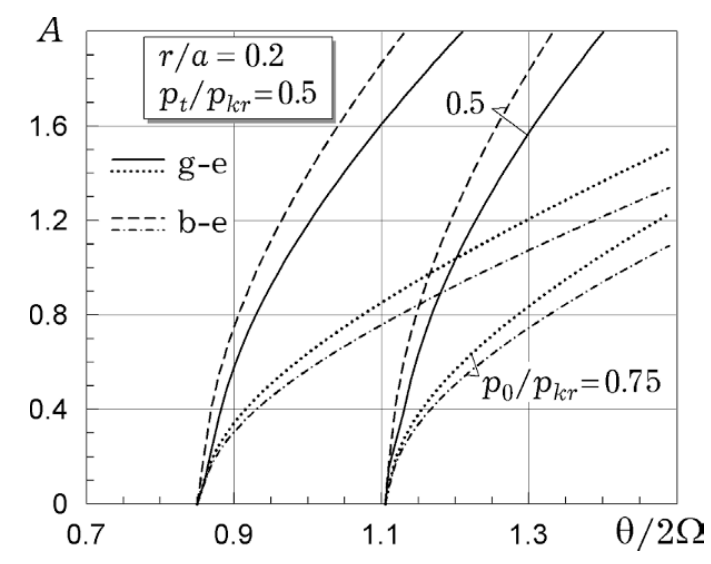

Fig. 10

To analyze nonlinear vibrations of the plate in the zone of the main parametric resonance, we plot the amplitude-frequency characteristics (AFC) for different loads (Figs. 8 and 9 for the boron-epoxy material) and different materials (Fig. 10). In Fig. 8, we show the amplitude-frequency dependences obtained for fixed values of the parameters $r / a=0.1, p_{0} / p_{k r}=0.25$, and $\varepsilon=0$ for different $p_{t} / p_{k r}=0.25,0.5,0.75$. The obtained re- 


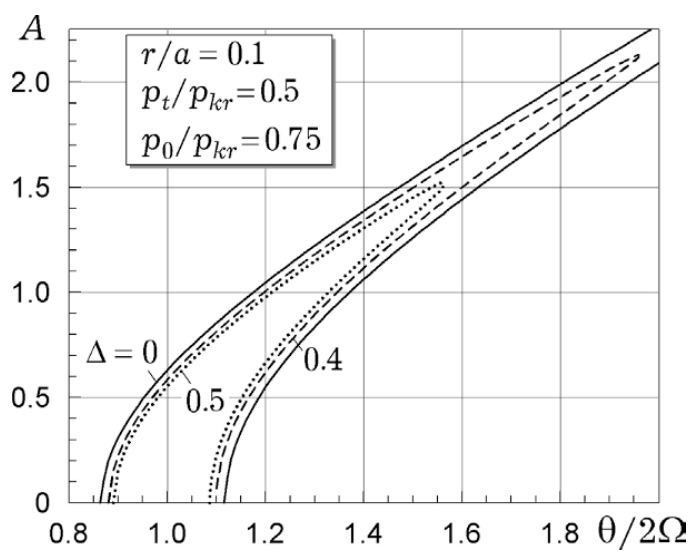

Fig. 11

sults enable us to conclude that, as the amplitude of the variable part of the load $p_{t}$ decreases, the branches of the AFC approach each other.

The results of investigation of the influence of the constant component of the load on the AFC are shown in Fig. 9 for the boron-epoxy material (for fixed $r / a=0.1, p_{t} / p_{k r}=0.5$, and $\varepsilon=0$ and different $p_{0} / p_{k r}=$ $0.25,0.5,0.75$ ) and in Fig. 10 for the glass-epoxy and boron-epoxy materials (for fixed $r / a=0.2$, $p_{t} / p_{k r}=0.5$, and $\varepsilon=0$ and different $\left.p_{0} / p_{k r}=0.25,0.75\right)$. The variations of the constant component of the load affect the slope of the curves and, at the same time, the influence is more pronounced for the plate made of the boron-epoxy material.

In Fig. 11, we present the amplitude-frequency dependences obtained for different values of the damping decrement.

\section{CONCLUSIONS}

In the present work, we propose a numerical-analytic approach to the investigation of parametric vibrations and stability of multilayer plates with symmetric structure and any form in the plan for different types of boundary conditions and a way of stacking the layers. The approach is based on the $R$-function theory and variational methods.

We obtain analytic expressions for finding the coefficients of ODEs obtained by reducing the original system with the use of multimode approximation of unknown functions. The algorithm is realized within the framework of the POLE-RL system. By using the developed software, we performed testing and solved new problems of vibration of multilayer complex-shaped plates under the action of static and periodic loads in the middle plane. We studied rectangular plates with an aim of comparing the values of the frequency ratio with the available data. The difference between the accumulated results and the data obtained in [12] does not exceed $3 \%$. We investigated vibrations of a multilayer plate of complex shape in the zone of the main parametric resonance and established the dependences of the analyzed characteristics (regions of dynamic instability and amplitude-frequency dependences) on the loading parameters, material of the plate, and its geometry.

\section{REFERENCES}

1. S. A. Ambartsumyan, Theory of Anisotropic Plates [in Russian], Nauka, Moscow (1987).

2. V. V. Bolotin, The Dynamic Stability of Elastic Systems, Holden-Day, San Francisco (1964). 
3. L. V. Kurpa, Method of R-Functions for the Solution of Linear Problems of Bending and Vibrations of Shallow Shells [in Russian], "KhPI" National Technical University, Kharkov (2009).

4. L. Kurpa and O. Mazur, "Parametric vibrations of plates of complex form in the plan," Mashynoznavstvo, No. 3 (129), 9-15 (2008).

5. V. L. Rvachev and L. V. Kurpa, R-Functions in Problems of the Theory of Plates [in Russian], Naukova Dumka, Kiev (1987).

6. J. Awrejcewicz, L. Kurpa, and O. Mazur, "Research of stability and nonlinear vibration by $R$-functions method," in: J. Awrejcewicz (editor), Modeling, Simulation, and Control of Nonlinear Engineering Dynamical Systems, Springer (2009), pp. 179-189.

7. S. Dash, A. V. Asha, and S. K. Sahu, "Stability of laminated composite curved panels with cutout using finite element method," in: Proc. of the 3rd Internat. Conf. on Theoretical, Applied, Computational and Experimental Mechanics (ICTACEM-2004) (December 28-31, 2004), 2004 IIT, Kharagpur; http://hdl.handle.net/2080/316.

8. M. P. Nemeth, Buckling and Postbuckling Behavior of Laminated Composite Plates with a Cutout. NASA Technical Paper 3587 (1996).

9. T. Y. Ng, K. Y. Lam, and J. N. Reddy, "Dynamic stability of cross-ply laminated composite cylindrical shells," Int. J. Mech. Sci., 40, No. 8, 805-823 (1998).

10. S. K. Sahu and P. K. Datta, "Research advances in the dynamic stability behavior of plates and shells: 1987-2005, Part 1: Conservative system," Appl. Mech.Rev., 60, No. 2, 65-75 (2007).

11. G. J. Simitses, "Instability of dynamically loaded structures," Appl. Mech.Rev., 40, No. 10, 1403-1408 (1987).

12. M. K. Singha and R. Daripa, "Nonlinear vibration and dynamic stability analysis of composite plates," J. Sound Vibrat., 328, No. 4, 541-554 (2009). 